\title{
MORSE INEQUALITIES FOR A DYNAMICAL SYSTEM
}

\author{
BY STEPHEN SMALE ${ }^{1}$
}

Communicated by S. Eilenberg, October 24, 1959

1. Introduction. We consider dynamical systems $(X, M)$, where $X$ is a $C^{\infty}$ vector field on a $C^{\infty}$ closed manifold $M$ satisfying the following conditions.

(1) There are a finite number of singular points of $X$, say $\beta_{1}, \cdots, \beta_{k}$, each of simple type. This means that at each $\beta_{i}$, the matrix of first partial derivatives of $X$ in local coordinates has eigenvalues with real part nonzero.

(2) There are a finite number of closed orbits (i.e., integral curves) of $X$, say $\beta_{k+1}, \cdots, \beta_{m}$, each of simple type. This means that no characteristic exponent (see, e.g., [2]) of $\beta_{i}, i>k$, has absolute value 1 .

(3) The limit points of all the orbits of $X$ as $t \rightarrow \pm \infty$ lie on the $\beta_{i}$. In other words, denote by $\phi_{t}$ the 1-parameter group of transformations generated by $X$ (as we do throughout this paper). Let

$$
\alpha(y)=\underset{t \rightarrow-\infty}{\operatorname{limit} \operatorname{set}} \phi_{t}(y), \quad \omega(y)=\underset{t \rightarrow \infty}{\operatorname{limit} \operatorname{set}} \phi_{t}(y), \quad y \in M .
$$

Then for each $y, \alpha(y)$ and $\omega(y)$ are contained in the union of the $\beta_{i}$.

(4) The stable and unstable manifolds of the $\beta_{i}$ (see $\$ 2$ for the definition) have normal intersection with each other. More precisely for each $i$ let $W_{i}$ be the unstable manifold and $W_{i}^{*}$ the stable manifold of $\beta_{i}$ and for $x \in W_{i}$ (or $W_{i}^{*}$ ) let $W_{i x}$ (or $W_{i x}^{*}$ ) be the tangent space of $W_{\boldsymbol{i}}$ (or $W_{\boldsymbol{i}}^{*}$ ) at $x$. Then for each $i, j$ if $x \in W_{\mathbf{i}} \cap W_{j}^{*}$,

$$
\operatorname{dim} W_{i}+\operatorname{dim} W_{j}^{*}-n=\operatorname{dim}\left(W_{i x} \cap W_{j x}^{*}\right) .
$$

See [5] for example for more details.

(5) If $\beta_{i}$ is a closed orbit there is no $y \in M$ with $\alpha(y)=\omega(y)=\beta_{i}$.

First we remark that systems satisfying (1)-(5) may be very important because of the following possibilities.

(A) It seems at least plausible that systems satisfying (1)-(5) form an open dense set in the space (with the $C^{1}$ topology) of all vector fields on $M$.

(B) It seems likely that conditions (1)-(5) are necessary and sufficient for $X$ to be structurally stable in the sense of Andronov and Pontrjagin [1]. See also [6]. [9].

(A) and (B) have been proved for the case $M$ is a 2-disk, [3] and

${ }^{1}$ Supported by a National Science Foundation Postdoctoral Fellowship. 
We expect to have more to say about this subject at another time.

It is true that conditions (1)-(5) are independent.

With $(X, M)$ as above let $\sigma_{i}=\operatorname{dim} W_{i}$. Then if $i \leqq k, \sigma_{i}$ is the number of eigenvalues associated to $\beta_{i}$ with real part positive. Let $a_{q}$ be the number of $\beta_{i}, i \leqq k$ with $\sigma_{i}=q$. If $i>k, \sigma_{i}$ is one more than the number of characteristic exponents of $\beta_{i}$ with absolute value greater than one. Let $b_{q}$ be the number of $\beta_{i}, i>k$, with $\sigma_{i}=q$.

The main goal of this paper is to prove

Theorem 1.1. Let $(X, M)$ be a system satisfying (1)-(5), $K$ any field, $R_{q}$ the rank of $H^{q}(M, K)$, and $M_{q}=a_{q}+b_{q}+b_{q+1}$. Then $M_{q}$ and $R_{Q}$ satisfy the Morse relations

$$
\begin{aligned}
M_{0} & \geqq R_{0}, \\
M_{1}-M_{0} & \geqq R_{1}-R_{0}, \\
M_{2}-M_{1}+M_{0} & \geqq R_{2}-R_{1}+R_{0}, \\
\ldots & \ldots \ldots \\
\sum_{k=0}^{n}(-1)^{k} M_{k} & =(-1)^{n} \chi
\end{aligned}
$$

where $\operatorname{dim} M=n$ and $\chi$ is the Euler characteristic of $M$ with respect to $K$.

Theorem 1.1 contains the Theorem of El'sgol'c [4] which excludes closed orbits. It also contains Reeb's theorem [11] which excludes singular points. However, both El'sgol'c and Reeb made the highly restrictive assumption ${ }^{2}$ that no orbit joined saddle points (i.e., $\beta_{i}$, $i \leqq k$ with $\left.\sigma_{i} \neq 0, n\right)$ or saddle type closed orbits (i.e., $\beta_{i}, i>k$ with $\left.\sigma_{i} \neq 1, n\right)$.

Also it follows from the following theorem which we prove elsewhere that Theorem 1.1 includes the classical theorem of Morse [8] for a function $f$ on $M$ with nondegenerate critical points.

Theorem 1.2. If $X=\operatorname{grad} f, f a C^{\infty}$ function on $M$ with nondegenerate critical points, then $X$ can be $C^{1}$ approximated by a $C^{\infty}$ field $Y$ on $M$ such that $(Y, M)$ satisfies (1)-(5) with no closed orbits.

2. Construction of the stable and unstable manifolds. 2.1. Suppose $\beta$ is a singular point of simple type of the $C^{\infty}$ system $(X, M)$. Let $k$ be the number of eigenvalues associated to $\beta$ with real part positive. Then (e.g., [2, p. 330]) there is a $k$ dimensional $C^{\infty}$ submanifold $W$ of $M$ passing through $\beta$ such that if $x \in W$ then $\alpha(x)=\beta$. If $k=0$, let

2 Reeb has asked me to note that his footnote 3,2 nd paragraph, of $[11$, p. 62] (that this assumption is unnecessary) is incorrect 
$W=\beta$. Then $W$ is tangent at $\beta$ to the linear subspace of the tangent space $M_{\beta}$ of $M$ at $\beta$ defined by these $k$ eigenvalues [2, p. 333]. $W$ is called the unstable manifold of $X$ at $\beta$. Let $R^{k}$ denote Euclidean $k$ space considered as a vector space. We will show that $W$ is the image of a continuous 1-1 onto map $f: R^{k} \rightarrow W$, with $f(0)=\beta$, and $f$ is $C^{\infty}$ with Jacobian of rank $k$ except at 0 . Consider the new system $X^{*}$ obtained by reversing the direction of each vector of $X$ on $M$. Then $\beta$ is a simple singularity of $X^{*}$ and the above applies to yield the unstable $(n-k)$-dimensional manifold $W^{*}$ of $X^{*}$ at $\beta$. Call $W^{*}$ the stable manifold of $X$ at $\beta$. Note $W$ and $W^{*}$ have normal intersection at $\beta$.

2.2. Suppose $\beta$ is a closed orbit of $(X, M)$ of simple type. Let $k-1$ be the number of characteristic exponents of $\beta$ with absolute value greater than one. Then ([7] or [13]) there is a $k$-dimensional $C^{\infty}$ submanifold $W$ of $M$ passing through $\beta$ such that if $x \in W$ then $\alpha(x)=\beta$. If $k=1$ let $W=\beta$. Also $W$ is tangent at each point $y$ of $\beta$ to the linear subspace of $M_{y}$ defined by these $k-1$ characteristic exponents and the tangent vector of $\beta$ at $y$. Call $W$ the unstable manifold of $X$ at $\beta$. We will show there is a continuous 1-1 onto map $f: R^{k-1} \times S^{1} \rightarrow W$, with $f\left(0 \times S^{1}\right)=\beta$, and except along $0 \times S^{1}$ is $C^{\infty}$ with Jacobian of rank $k$. Similarly to 2.1 , one defines the stable manifold $W^{*}$ of $X$ at $\beta$ whose dimension in this case is $n-k+1$.

We now construct the map $f$ of 2.1 .

There exists ${ }^{3}$ a differentiably imbedded $(k-1)$-sphere $K$ in $W$, which is everywhere transversal to $X$. Let $S_{0}$ be the unit sphere of $R^{k}$ and $h: S_{0} \rightarrow K$ be a diffeomorphism. (A diffeomorphism is $C^{\infty}$ homeomorphism with a differentiable inverse.) Let $\psi_{t}$ be the 1parameter group of transformations of $R^{k}$ generated by the vector field $Y(x)=x$ on $R^{k}$. For $x \in R^{k}, x \neq 0$, let $t(x)$ be the unique $t$ such that $x /\|x\|=\psi_{t(x)}(x) \in S_{0}$. Then let $f(0)=\beta$ and $f(x)=\phi_{-t(x)} h \psi_{t(x)}(x)$. It is easy to check that $f: R^{k} \rightarrow W$ thus defined has the desired properties.

To construct the map $f: R^{k-1} \times S^{1} \rightarrow W$ of 2.2 , first let $Y$ be the vector field $(x, 1)$ on $R^{k-1} \times S^{1}$. Then if $\psi_{t}$ is the 1-parameter group of transformations generated by $Y$ we have $\psi_{t}(x, 0)=\left(x e^{t}, t \bmod 2 \pi\right)$. Let $R^{k-1}=R^{k-1} \times 0 \subset R^{k-1} \times S^{1}$, and $C$ be the unit ball in $R^{k-1}, \partial C=S_{0}$. Define $q: R^{k-1} \rightarrow R^{k-1}$ by $q(x)=x e^{2 \pi}$ and let $q^{i} S_{0}=S_{i}$ for each integer $i$.

Let $Q$ be a surface of section (i.e., transversal to $X$, see [6]) locally about a point of $\beta$ in $W$, diffeomorphic to a $(k-1)$-cell. Then [6] the orbits of $X$ define a diffeomorphism $h: Q \rightarrow Q$ in a neighborhood of $\beta \cap Q$ leaving $\beta \cap Q$ fixed. There is ${ }^{3}$ a closed $k$-cell $B$ differentiably

By Liaponov theory for example. 
imbedded in $Q, \partial B=F_{0}$ such that $h^{-1}\left(F_{0}\right)$ is contained in the interior of $B$. Let $h^{i}\left(F_{0}\right)=F_{i}, i \leqq 0$.

Let $f$ be an orientation preserving diffeomorphism of a neighborhood $V_{0}$ of $S_{0}$ in $R^{k-1}$ into a neighborhood of $F_{0}$ in $Q$. Then extend $f$ to a neighborhood of $U_{i \leq 0} S_{i}$ in $R^{k-1}$ into a neighborhood of $U_{i \leq 0} F_{i}$ in $Q$ by the formula

$$
f(x)=h^{-i} f q^{i}(x), \quad x \in \operatorname{nbd} . V_{i} \text { of } S_{i} .
$$

This makes sense for an appropriate choice of the $V_{i}$ 's. Now consider the closed region $U$ in $R^{k-1}$ bounded by $S_{0}$ and $S_{-1}$. We have defined $f$ in a neighborhood of the boundary $\partial U$ of $U$. After restricting $f$ to a smaller neighborhood of $\partial U, f$ can be extended to a diffeomorphism of all of $U$ into the region of $Q$ bounded by $F_{0}$ and $F_{-1}$. This fact follows from arguments which are now standard in differential topology. We won't include them here. Then as in 2.3 we can extend $f$ to a map of all of $C$ into $B$ which is a diffeomorphism except at $f(0)=\beta \cap Q$.

Next define $f$ on $P=\left\{\psi_{t}(x) \mid x \in C, t<0\right\}$ by the following: Let $\tau(x, \theta)$ be the smallest positive number such that $\phi_{r(x, \theta)} f \psi_{-\theta}(x, \theta)$ has $\theta$ as its second coordinate in a fixed product structure $Q \times \beta,(x, \theta) \in P$. Then let $f(x, \theta)=\phi_{\tau(x, \theta)} f \psi_{-\theta}(x, \theta)$. Define $f: 0 \times S^{1} \rightarrow \beta$ by $f(0 \times \theta)=\theta$.

Consider now the surface of section $S_{-1} \times S^{1}=A$ in $R^{k-1} \times S^{1}$ and its image under $f$. Restrict $f$ to the closure of the bounded component $K$ of $A$. Finally extend $f$ to all of $R^{k-1} \times S^{1}$ as follows. For $y \in R^{k-1}$ $\times S^{1}-K$ let $t(y)$ be the unique $t$ such that $\psi_{t(y)}(y) \in A$. Then let $f(y)=\phi_{-t(y)} f \psi_{t(y)}(y)$. After a change of parameter near $A, f$ will have our desired properties.

3. Implications of (1)-(5). Assume throughout this section that $(X, M)$ is given as in $\$ 1$. If $\beta_{i}$ is a singular point then $f_{i}: R^{k} \rightarrow W_{i}$ is as in 2.1. If $\beta_{i}$ is a closed orbit then $f_{i}: R^{k-1} \times S^{1} \rightarrow W_{i}$ is as in 2.2.

Lemma 3.1. If $x \in M, \alpha(x)=\beta_{i}, \omega(x)=\beta_{j}$, then $\operatorname{dim} W_{i} \geqq \operatorname{dim} W_{j}$ and equality can occur only if $\beta_{j}$ is a closed orbit.

Proof. Clearly $x \in W_{i} \cap W_{j}^{*}$ and by (4) we have that $\operatorname{dim} W_{i}$ $+\operatorname{dim} W_{j}^{*}-n \geqq 1$. But $\operatorname{dim} W_{j}^{*}=n-\operatorname{dim} W_{j}$ if $\beta_{j}$ is a singular point and $\operatorname{dim} W_{j}^{*}=n-\operatorname{dim} W_{j}+1$ if $\beta_{j}$ is a closed orbit. Then 3.1 follows.

See $[12]$ for the following.

Lemma 3.2. Suppose $W_{i} \cap W_{j}^{*} \neq \varnothing \varnothing$ and $x \in W_{j}$. Then there exists a cell neighborhood $H$ of $x$ in $W_{j}$ such that given $\delta>0$, there is $a y \in W_{i}$ with $d(x, y)<\delta$ and if $\operatorname{dim} W_{i}=\operatorname{dim} W_{j}$, there is a subcell $K$ of $W_{i}$ such that $H$ and $K$ are within $\delta$ in a $C^{1}$ metric. 
Define $\partial W_{j}=\left\{\lim _{k \rightarrow \infty} f_{j}\left(x_{k}\right) \mid x_{k}\right.$ any sequence in $R^{k}$ with no lps. $\}$. Then let $\partial^{2} W_{j}=\partial\left(\partial W_{j}\right)$, etc. Note $\mathrm{Cl} W_{i}=W_{i} \cup \partial W_{i}$.

Lemma 3.3. If $W_{i} \cap W_{j}^{*} \neq \varnothing, \partial W_{i} \supset W_{j}$.

This follows from 3.2.

Lemma 3.4. Suppose $\operatorname{dim} W_{i}=\operatorname{dim} W_{k}=\operatorname{dim} W_{j}$. If $W_{i} \cap W_{k}^{*} \neq \varnothing$ and $W_{k} \cap W_{j}^{*} \neq \varnothing$ then $W_{i} \cap W_{j}^{*} \neq \varnothing$.

Proof. Let $x \in W_{k} \cap W_{j}^{*}$; apply 3.2 using the fact that $W_{i} \cap W_{k}^{*}$ $\neq \varnothing$. Since $W_{k}$ and $W_{j}^{*}$ have normal intersection at $x$, it follows from 3.2 that $W_{i} \cap W_{j}^{*} \neq \varnothing$.

Lemma 3.5. Suppose $W_{i_{k}} \cap W_{i_{k+1}}^{*} \neq \varnothing, k=1, \cdots$, m. Then $W_{i_{k}}$ $\neq W_{i_{j}}$ if $j \neq k$.

Proof. First note by $3.1, \operatorname{dim} W_{i_{k+1}} \leqq \operatorname{dim} W_{i_{k}}$ and equality occurs only if $\beta_{i_{k+1}}$ is a closed orbit. This implies we can restrict ourselves to the case of the lemma where all the $W_{i_{k}}$ 's are of the same dimension. Then if $W_{i_{k}}=W_{i_{j}}, k \neq j, 3.4$ implies that $W_{i_{k}} \cap W_{i_{j}}^{*} \neq \varnothing$. This contradicts condition (5).

LEMma 3.6. If $\partial W_{\gamma} \cap W_{\delta} \neq \varnothing$, then there is a sequence $W_{i_{1}}, \cdots, W_{i_{m}}$ such that $W_{i_{k}} \cap W_{i k+1}^{*} \neq \varnothing, W_{\gamma}=W_{i_{1}}$, and $W_{\delta}=W_{i_{m}}$.

Proof. Let $\alpha\left(W_{\delta}^{*}\right)=\lim _{t \rightarrow-\infty} W_{\delta}^{*}$. Then it follows that $\mathrm{Cl} W_{\gamma}$ $\cap \alpha\left(W_{\delta}^{*}\right) \neq \varnothing$. Let $\beta_{j} \in \mathrm{Cl} W_{\gamma} \cap \alpha\left(W_{\delta}^{*}\right)$. Then $W_{j} \cap W_{\delta}^{*} \neq \varnothing$. If $j \neq \gamma$, similarly let $\beta_{k} \in \mathrm{Cl} W_{\gamma} \cap \alpha\left(W_{j}^{*}\right)$. Induction and 3.5 yield 3.6.

Lemma 3.7. If $\partial W_{i} \cap W_{j} \neq \varnothing$, then $\partial W_{i} \supset W_{j}$ and either $\operatorname{dim} W^{i}$ $>\operatorname{dim} W_{j}$ or $\operatorname{dim} W_{i}=\operatorname{dim} W_{j}, W_{i} \cap W_{j}^{*} \neq \varnothing$, and $\beta_{j}$ is a closed orbit

This follows from 3.6, 3.5, 3.3, 3.1, and 3.4.

Lemma 3.8. Each $W_{i}$ is an imbedded $R^{p}$ or $R^{p-1} \times S^{1}$.

This follows from $\$ 2,3.7$ and (5).

Lemma 3.9. $\partial^{k} W_{i} \neq \varnothing$, any $i$ for large enough $k$.

If not there is a $W_{j}$ such that $\partial^{m} W_{j} \cap W_{j} \neq \varnothing$. By 3.7 then $\partial W_{j} \cap W_{j}$ $\neq \varnothing$, contradicting 3.8 .

4. On Morse theory. A version of one of the standard theorems of Morse theory is stated in this section. The proof is a short wellknown argument using the exact cohomology sequence of a pair and for example can be found in [10].

TheOREM 4.1. Let $M$ be an n-dimensional topological space with closed subspace $L^{p}$ for each integer $p$ such that $L^{p} \supset L^{p-1}$, and there 
exist integers $a, b$ with $L^{a}=\varnothing$ and $L^{b}=M$. Using any fixed cohomology theory and coefficient field, assume dimension $H^{q}\left(L^{p}, L^{p-1}\right)$ is finite for each $p$ and $q$. Let $B_{q}=\operatorname{dim} H^{q}(M)$ and $M_{q}=\sum_{r=a}^{b} \operatorname{dim} H^{q}\left(L^{r}, L^{r-1}\right)$. Then $M_{q}$ and $B_{q}$ satisfy the Morse relations

$$
\begin{gathered}
M_{0} \geqq B_{0}, \\
M_{1}-M_{0} \geqq B_{1}-B_{0}, \\
M_{2}-M_{1}+M_{0} \geqq B_{2}-B_{1}+B_{0}, \\
\cdot \cdot \cdot \cdot \cdot \cdot \cdot \cdot \cdot \cdot \cdot \cdot \cdot \cdot k \\
\sum_{k=0}^{n}(-1)^{k} M_{k}=\sum_{k=0}^{n}(-1)^{k} B_{k} .
\end{gathered}
$$

5. Proof of the main theorem. Define $K^{p}=\bigcup_{\mathrm{dim} W_{i \leq p}} W_{i}$. Thom in [14] considers subspaces related to $K^{p}$ to prove the classical Morse inequalities. By 3.7 it follows that the $K^{p}$ are closed sets. However, examples show that $K^{p}$ has the following bad property. It may be that for $W_{i} \subset K^{p}, \partial W_{i}$ is not contained in $K^{p-1}$. To avoid this we define a new structure on $M$.

We define by induction a sequence of closed subsets $L_{i}$ of $M$ with $L_{i} \supset L_{i-1}$ and $L_{i}=M$ for large enough $i$. Define $L_{0}=\varnothing$, and if $L_{i-1}$ has been defined, let $L_{i}$ be the union of all the $W_{j}$ whose boundary lies in $L_{i-1}$. It is immediate that $L_{i} \supset L_{i-1}$, that $L_{i}$ is closed in $M$ and that $L_{i}-L_{i-1}$ consists of a disjoint union of $W_{j}$. It follows from 3.9 that there is an integer $b$ such that $L_{b}=M$.

One can construct an example to show that the $L_{i}$ need not be locally connected and that for $W_{0} \subset L_{i}-L_{i-1}, W_{1} \subset L_{i-1}, \operatorname{dim} W_{0}$ $=\operatorname{dim} W_{1}$.

LEmma 5.1. Using Cech theory if $M_{q}$ is as in 1.1 then

$$
M_{q}=\sum_{i=0}^{b} \operatorname{dim} H^{q}\left(L_{i}, L_{i-1}\right) .
$$

Proof. As noted previously $L_{i}-L_{i-1}$ consists of a disjoint union of the $W_{j}$ and as $i$ ranges from 0 to $b$ all the $W_{j}$ are obtained. Denoting cohomology with compact carriers by $K_{K}^{q}$, since $H_{K}^{q}(P-Q)=H^{q}(P, Q)$ for Cech theory, we have

$$
\sum_{i=0}^{b} \operatorname{dim} H^{q}\left(L_{i}, L_{i-1}\right)=\sum_{\mathrm{all} W_{j}} \operatorname{dim} H_{K}^{q}\left(W_{j}\right) .
$$

Using 3.8 and Poincaré duality $H_{K}^{q}\left(W_{j}\right)=H_{\text {dim } W_{j-q}}\left(W_{j}\right)$. Furthermore $\operatorname{dim} H_{0}\left(W_{j}\right)=1$ for all $j, \operatorname{dim} H_{1}\left(W_{j}\right)=1$ if $\beta_{j}$ is a closed orbit and $\operatorname{dim} H_{p}\left(W_{j}\right)=0$ otherwise. The lemma follows. 
Theorem 1.1 follows from 4.1 and 5.1.

6. An analogue of the main theorem. Suppose instead of a vector field $X$ on $M$, we just have given a $C^{\infty}$ diffeomorphism $h$ on $M$ which satisfies certain conditions analogous to (1)-(5).

$\left(1^{\prime}\right)$ There are a finite number of periodic points (i.e., $x \in M$ such that $h^{p}(x)=x$ for some integer $p$ ) of $h$ of simple type (i.e., the differential of $h$ at $p$ has no eigenvalue of absolute value 1 ).

$\left(3^{\prime}\right)$ The limit points of all the orbits of $h$ (i.e., $\left\{h^{p}(x) \mid\right.$ all integers $p\}=$ orbit of $x$ ) are periodic points.

$\left(4^{\prime}\right)$ The "stable" and "unstable" manifolds of the periodic points have normal intersection.

The previous theory extends to cover this case. In particular if $M_{q}$ is the number of periodic points with $q$ eigenvalues having absolute value greater than one, the Morse relations of 1.1 hold.

One can ask the corresponding questions of (A) and (B) of $\S 1$ for the above situation.

\section{REFERENCES}

1. A. A. Andronov and L. S. Pontrjagin, Systemes grossiers, Dokl. Akad. Nauk vol. 14 (1937) pp. 247-250.

2. E. A. Coddington and N. Levinson, Theory of ordinary differential equations, New York, McGraw-Hill, 1955.

3. H. De Baggis, Dynamical systems with stable structure, Contributions to the Theory of Nonlinear Oscillations, Princeton University Press, vol. 2, 1952, pp. 37-59 (Annals of Mathematics Studies, no. 29).

4. L. E. El'sgol'c, An estimate for the number of singular points of a dynamical system defined on a manifold, Amer. Math. Soc. Translations, no. 68, 1952. Translated from Mat. Sb. (N.S.) vol. 26 (68) (1950) pp. 215-223.

5. R. K. Lashof and S. Smale, Self-intersections of immersed manifolds, J. Math. Mech. vol. 8 (1959) pp. 143-158.

6. S. Lefschetz, Differential equations: geometric theory, New York, Interscience Publishers, 1957.

7. D. C. Lewis, Invariant manifolds near an invariant point of unstable type, Amer. J. Math. vol. 60 (1938) pp. 577-587.

8. M. Morse, Calculus of variations in the large, Amer. Math. Soc. Colloquium Publications, vol. 18, 1934.

9. M. Peixoto, On structural stability, Ann. of Math. vol. 69 (1959) pp. 199-222.

10. E. Pitcher, Inequalities of critical point theory, Bull. Amer. Math. Soc. vol. 64 (1958) pp. 1-30.

11. G. Reeb, Sur certaines proprietes topologiques des trajectoires des systèmes dynamiques, Acad. Roy. Belg. Cl. Sci. Mém. Coll. in $8^{\circ} 27$ no. 9 (1952).

12. S. Smale, On structural stability, to appear.

13. S. Sternberg, Local contractions and a theorem of Poincare, Amer. J. Math. vol. 79 (1957) pp. 809-724.

14. R. Thom, Sur une partition en cellules associee d une fonction sur une variete, C. R. Acad. Sci. Paris vol. 228 (1949) pp. 973-975.

Institute for AdVANCEd Study 